\title{
Masking of the signal for lateralization of tones
}

\author{
W. A. WILBANKS \\ University of Georgia, Athens, Georgia 30602
}

\begin{abstract}
Elliott and Wilbanks (1980) have shown that when listeners attempt to localize a tone in noise, the "signal" for lateralization is the change in the interaural phase between tone plus noise and the interaural phase of the noise alone. This "signal" can be masked by increasing the level of the noise and by reducing the interaural correlation of the noise. Data are presented showing how the masking of the signal for lateralization varies as a function of noise correlation and tone frequency. The results are interpreted in terms of the vector model of detection and localization.
\end{abstract}

When listeners attempt to localize a tone in noise, the "signal" for localization is the change in the interaural phase between the signal plus noise at the two ears and the interaural phase of the noise alone. Elliott and Wilbanks (1980) have shown that this signal can be masked, resulting in a decrement in lateralization performance, by increasing the level of the noise and, more dramatically, by reducing the interaural correlation of the noise. In that study, a $250-\mathrm{Hz}$ tone was presented binaurally at a level of $74 \mathrm{db}$ SPL. A wide-based noise was presented at two overall levels, $+64 \mathrm{~dB}$ and $+84 \mathrm{~dB}$ SPL. The interaural correlation of the noise was varied between unity and zero. With an 84-dB noise, the interaural phase difference for $85 \%$ correct lateralizations was found to increase from about $7 \mathrm{deg}$ to $21 \mathrm{deg}$ as the noise correlation was reduced from unity to zero. Although the same trend was evident in the low-noise condition $(64 \mathrm{~dB})$, the effect of reducing noise correlation was considerably less. In this condition, the interaural phase difference for $85 \%$ correct choices increased from about $5 \mathrm{deg}$ to $10 \mathrm{deg}$ as the noise correlation was reduced from unity to zero. Under conditions presumably minimizing the effects of noise, Zwislocki and Feldman (1956) found that interaural phase differences on the order of 2 deg can be detected as a shift in the location of the sound image. It would appear that the low-level correlation noise used by Elliott and Wilbanks (1980) only slightly masked the signal for laterlization. In the present study, we extend our earlier work on the masking of the signal for lateralization of tone to a wider range of tonal frequencies.

\section{METHOD}

Three tonal signals were used: $500 \mathrm{~Hz}, 750 \mathrm{~Hz}$, and $1,000 \mathrm{~Hz}$. Each tone was presented binaurally to the listener at a level of $74 \mathrm{~dB}$ re. .0002 microbar. The interaural phase of the tone was

Correspondence should be sent to W. A. Wilbanks, Department of Psychology, University of Georgia, Athens, Georgia 30602 . varied using a Grason-Stadler phase shifter. The interaural phase value for a given condition was determined by measuring the corresponding interaural time difference with an Atec electronic counter. The duration of the tone $(150 \mathrm{msec})$ was controlled by an electronic clock that triggered a Grason-Stadler switch, gating the tone with a $10-\mathrm{msec}$ on-off time. The level of the signal was measured across the subjects' earphones (TDH-49) with a true-RMS voltmeter and a wave analyzer.

Each experimental session consisted of 100 two-alternative forced-choice trials. The sequence of events within each trial was: (1) warning interval, $100 \mathrm{msec}$, (2) pause, $800 \mathrm{msec}$, (3) first observation interval, $150 \mathrm{msec}$, (4) pause, $800 \mathrm{msec}$, (5) second observation interval, $150 \mathrm{msec}$, and (6) response period, 2 sec. The occurrence of the warning and observation intervals was marked for the observer by lights. The leading channel of the output of the phase shifter was electronically commutated so that it could be presented to either the left or right ear. Two types of trials occurred during each 100-trial session: Type 1-(a) signal leading in phase at the left ear during the first observation interval, (b) signal leading in phase at the right ear during the second observation interval. A Type 2 trial consisted of the reverse sequence of events. After each trial, the observer's task was to state which type of trial had occurred. The a priori probability of each type was .50 .

The signals were presented in a background of wide-band noise, cut off at the lower end by response of the earphones and at the upper end by a 3,000-Hz low-pass passive filter. The noise was presented at an overall level of $+84 \mathrm{~dB}$ SPL re. .0002 microbar. Two noise generators were used to obtain noise with positive interaural correlations of $+1.00,+.87,+.50,+.22$, and zero. The perfect positive correlation (NO) was achieved by using a single noise source for both ears. The zero correlation (NU) was achieved by using independent noise generators, one for each ear. Interaural correlations between unity and zero were obtained by mixing the outputs of the two noise sources in the channel to one ear, the noise at the other ear being supplied by only one of the generators. In this case, the interaural correlation is given by: $\left.\mathrm{r}^{2}=\mathrm{e}_{\mathrm{c}}{ }^{2} / \mathrm{e}_{\mathrm{c}}{ }^{2}+\mathrm{e}_{\mathrm{u}}{ }^{2}\right)$, where $\mathrm{e}_{\mathrm{c}}$ is the RMS voltage common to both ears, and $e_{u}$ is the RMS voltage of the noise unique to one ear. The values of $e_{c}^{2}$ and $\left(e_{c}{ }^{2}+e_{u}{ }^{2}\right)$ for any given correlation were equal. This way of varying interaural correlation has been thoroughly discussed by Jeffress and Robinson (1962) and Licklider and Dzendolet (1948).

Four observers were used. Since all had had considerable experience with detection and lateralization tasks, no special instructions were required, and no system of trial-by-trial feedback was used. The observers were sufficiently trained so that no correction for response bias was required. All listening was done in standard IAC booths. For each experimental condition, a 
minimum of five values of interaural phase for the $250-\mathrm{Hz}$ tone were used, and at least 500 forced-choice trials were given for each value of interaural phase for the tone. From these data, psychometric functions were obtained relating percentage correct choices to interaural phase value. From these psychometric functions, the main dependent variable was determined: the interaural phase for the tone for $85 \%$ correct lateralization choices.

\section{RESULTS AND DISCUSSION}

The results are given in Table 1 . Here, mean performance of the four subjects with $500-\mathrm{Hz}, 750-\mathrm{Hz}$, and $1,000-\mathrm{Hz}$ tones are shown as a function of noise correlation. Data from our previous study for $250 \mathrm{~Hz}$ are included for comparison (Elliott \& Wilbanks, 1980).

As mentioned previously, Zwislocki and Feldman (1956) found that, under conditions presumably minimizing the effects of noise, interaural phase differences for tone on the order of $2 \mathrm{deg}$ can be detected as a shift in the lateral position of the sound image. Examination of Table 1 shows clearly that as the level of noise is increased, the signal for lateralization, interaural phase, is masked. In order to maintain a constant level of lateralization performance, the interaural phase for tonal signals between $250 \mathrm{~Hz}$ and $1,000 \mathrm{~Hz}$ must be increased. With a noise correlation of unity, average interaural phase difference in degrees for $85 \%$ correct lateralization judgments must be increased to approximately $7 \mathrm{deg}$ for a $250 \mathrm{-Hz}$ signal, $8 \mathrm{deg}$ for the $500-\mathrm{Hz}$ signal, $10 \mathrm{deg}$ for the $750-\mathrm{Hz}$ signal, and about $14 \mathrm{deg}$ for a signal of $1,000 \mathrm{~Hz}$. As the correlation of the noise is reduced from unity to zero, the masking of the "signal" for lateralization dramatically increases for all tonal frequencies employed. This masking effect is more pronounced as the frequency of the signal is raised for all values of noise correlation.

These results clearly show that when a listener attempts to lateralize a tonal signal in a background of noise, two of the dominant variables determining the detectability of the signal for lateralization, interaural phase or time difference, are signal frequency and the interaural correlation for the noise.

Elliott and Wilbanks (1980) argued that the vector model of binaural detection proposed by Jeffress provides a convenient means of explaining the signal and noise interactions as to their effects on lateralization

Table 1

Average Interaural Phase Difference (in Degrees) for 85\% Correct Lateralization Judgments

\begin{tabular}{crrcc}
\hline & \multicolumn{4}{c}{ Tone Frequency (in Hertz) } \\
\cline { 2 - 5 } Noise & 250 & 500 & 750 & 1000 \\
\hline+1.00 & 7 & 8 & 10 & 14 \\
+.87 & 10 & 11 & 13 & 16 \\
+.50 & 17 & 18 & 20 & 22 \\
+.22 & 18 & 20 & 24 & 28 \\
.00 & 21 & 24 & 26 & 29 \\
\hline
\end{tabular}

performance (Jeffress, 1964, 1972; Jeffress, Blodgett, Sandel, \& Wood, 1956; Sandel, Teas, Fedderson, \& Jeffress, 1955; Jeffress, Note 1). We are still of the same opinion.

The model begins with an examination of the statistics of the noise. The band of noise that masks the tone can be described as a sinusoid that varies slowly in phase and in amplitude (monaural critical band), to which a signal of constant amplitude is added in random phase. The addition of the signal yields a signal-plusnoise resultant that will, in general, differ in phase from the original noise, sometimes advancing and sometimes retarding the noise in phase. If an out-of-phase signal is presented binaurally in a background of correlated noise, there will be an interaural phase difference between the signal plus noise and the noise alone. The size of the interaural phase shift will be determined, among other things, by the level of the signal relative to the level of the noise for any given phase relation at addition (i.e., signal-to-noise ratio, the interaural phase difference of the signal, and the correlation of the noise). Jeffress' model proposes that the basis for improved detection under conditions in which MLDs are obtained is the change in the correlation between the stimuli at the two ears when the signal is added to the noise. Even when a tone is completely detectable, as in the present study, the signal and noise must interact in a similar fashion. The lateral position of a low-frequency tone can be expected to depend on the phase relationships of the signal-plus-noise resultants at the two ears when the signal is added, rather than on the phase difference of the tone alone. In general, the higher the signal-tonoise ratio, and the larger the noise correlation, the more lateralization performance will depend upon the interaural phase of the tonal signal.

To summarize, if a tonal signal is presented in a background of noise, the listener must determine the lateral position of the signal-plus-noise resultant. With a tone of fixed intensity, the important variables determining lateralization performance are (1) interaural phase of the tone, (2) tone frequency, (3) signal-to-noise ratio, and (4) noise correlation. If a very low level of correlated noise is present, the interaural phase of the signalplus-noise resultant is determined primarily by the interaural phase of the tone. As the correlation of the noise is reduced from unity, the interaural phase of the tone must be increased in order to maintain a given level of lateralization performance. If a high level of correlated noise is present, the interaural phase of the tone must be significantly increased to maintain a constant level of performance. As the correlation of the noise is reduced, again, the interaural phase of the signal must be increased.

\section{REFERENCE NOTE}

1. Jeffress, L. A. Masking binaural phenomena (Report No. 245). Austin: University of Texas Defense Research Laboratory, 1965. 


\section{REFERENCES}

Elliott, T. F., \& Wilbanks, W. A. Lateralization of tonal signals in noise. Bulletin of the Psychonomic Society, 1980, 15 37-40.

JefFREss, L. A. Stimulus-oriented approach to detection. Journal of the Acoustical Society of America, 1964, 36, 766-774.

Jefrress, L. A. Binaural signal detection: Vector theory. In J. V. Tobias (Ed.), Foundations of modern auditory theory (Vol. 2). New York: Academic Press, 1972.

Jeffress, L. A., Blodgett, H. C., Sandel, T. T., \& Wood, C. L. Masking of tonal signals. Journal of the Acoustical Society of America, 1956, 28, 416-426.

Jefrress, L. A., \& Robinson, D. E. Formulas for the coefficient of interaural correlation for noise. Journal of the Acoustical Society of America, 1962, 34, 1658(L).

Licklider, J. C. R., \& Dzendolet, E. Oscillographic scatterplots illustrating various degrees of correlation. Science, 1948, 107, 121-124.

Sandel, T. T., Teas, D. C., Fedderson, W. E., \& Jeffress, L. A. Localization of sound from single and paired associates. Journal of the Acoustical Society of America, 1955, 27, 842-852.

Zwislocki, J., \& Feldman, R. S. Just noticeable differences in dichotic phase. Journal of the Acoustical Society of America, 1956, 28, 860-864.

(Received for publication January 10, 1983.) 